\title{
Primary Cutaneous Diffuse Large B-Cell Lymphoma
}

National Cancer Institute

\section{Source}

National Cancer Institute. Primary Cutaneous Diffuse Large B-Cell Lymphoma. NCI

Thesaurus. Code C45193.

A diffuse large B-cell lymphoma that arises from the skin. This category includes primary cutaneous diffuse large B-cell lymphoma, leg type, and primary cutaneous diffuse large B-cell lymphoma, other, which includes primary cutaneous intravascular large B-cell lymphoma and primary cutaneous T-cell/histiocyte-rich large B-cell lymphoma. 